\title{
Film Dosage Forms Prepared with Alginate for Oral Candidiasis Treatment
}

\author{
Yoshifumi Murata*, Honoka Kanemaru, Megumi Tsushima, Chieko Maida and Kyoko Kofuji \\ 1Faculty of Pharmaceutical Science, Hokuriku University, Japan \\ *Corresponding author: Yoshifumi Murata, Faculty of Pharmaceutical Science, Hokuriku University, Ho-3, Kanagawa-machi, Kanazawa 920-1181, \\ Japan, Tel: +81-76-229-6184; Fax: +81-76-229-2781; Email: y-murata@hokuriku-u.ac.jp
}

Submission: 悳 February 13, 2018; Published: 眥 March 20, 2018

\begin{abstract}
A film dosage form (FD) incorporating an antifungal agent, miconazole, was prepared with sodium alginate for the treatment of Candida infections. To ensure the safety of oral administration, FD was modified with additives, such as alginate hydrolysate, to control the drug dissolution rate. A soft film with a thickness of approximately $70 \mu \mathrm{m}$ was obtained and each FD was sufficiently strong to be applied by hand. FD immediately swelled in limited dissolution medium and then disintegrated to release the incorporated drug. The drug dissolution rate was controlled by the amount of additive in the film base solution. FD prepared with sodium alginate and the hydrolysate is useful for the treatment of oral candidiasis as the dosage form can be administered into the oral cavity to act directly on the affected site.
\end{abstract}

Keywords: Film dosage form; Sodium alginate; Alginate hydrolysate; Miconazole; Oral scandidiasis

\section{Introduction}

Alginic acid is an anionic polysaccharide that consists of $\alpha$-Lguluronic acid $(\mathrm{G})$ and $\beta$-D-mannuronic acid $(\mathrm{M})$. The ratio of the two uronic acids and the sequence is referred to as the block structure. Two types of block structure, G-block and M-block, were obtained by the hydrolysis of alginic acid. Sodium alginate (Alg-Na) is a water-soluble polysaccharide; recently, many species of Alg- $\mathrm{Na}$ have been widely utilized as a food or drug additive owing to the very low toxicity of Alg-Na after oral administration [1-3]. We have reported that the film dosage form (FD) could be prepared simply with polysaccharides [4]. In particular, a soft thin film is obtained through the utilization of high-molecular weight Alg-Na. Alg-Na is a useful base for the preparation of the FD, although low-molecular weight polymers do not form a uniform film.

When FDs prepared with water-soluble polymers, such as Alg$\mathrm{Na}$, are placed in a small amount of liquid, such as saliva, they quickly disintegrate and release the incorporated drug. The disintegration profiles of FDs differ between species [5]. FDs prepared with polysaccharides are useful for the treatment of localized problems in the oral cavity and to simplify the administration of drugs to patients [6]. Thus, FD is an excellent dosage form, especially for oral care. The drug release rate must be controllable by modification of FD in accordance with a purpose.

Oral candidiasis is a fungal infection caused by Candida strains. These microorganisms are not harmful to healthy individuals, but can cause infections in immune compromised patients. It is also a common disease in elderly persons or patients with decreased saliva secretion [7,8]. Miconazole nitrate (MCZ) is an antifungal agent used to treat Candida infections and is directly applied in the oral cavity to avoid the side effects such as cytochrome P450 inhibition [9]. MCZ gel preparations are used for the topical treatment of oral candidiasis; however, the application of the dosage form is a tedious procedure for patients and is difficult in elderly patients.

As the antifungal agent must be applied repeatedly to completely treat oral candidiasis, all materials used for FD preparation must be safe for use in the oral cavity and the amount of the drug that reaches the site per unit time should be controlled. We have tried to prepare FDs that incorporated MCZ and Alg-Na to control the drug dissolution rate [10]. In the present study, FDs were modified by additives, such as alginate hydrolysate, to control the MCZ dissolution rate in a limited dissolution medium.

\section{Experimental}

\section{Materials}

Alg-Na (300 cps, Kyoto, Japan) was obtained from Nacalai Tesque Inc. and a $1.5 \%$ Alg-Na solution prepared with deionized water was used as the film base. A low-molecular weight Alg-Na, ULV-L3, was supplied by Kimica Co. (Tokyo, Japan). $\mathrm{MCZ}\left(\mathrm{C}_{18} \mathrm{H}_{14} \mathrm{Cl}_{4} \mathrm{~N}_{2} \mathrm{O} \mathrm{HNO}_{3}\right.$, M.W. 479.14) was obtained from Wako Pure Chemicals (Osaka, Japan). All other chemicals were of reagent grade. 
Preparation of alginate hydrolysates (G-block, M-block) and their sodium salts Alg-Na was partially hydrolyzed $(0.2 \mathrm{M} \mathrm{HCl}$, $2 \mathrm{~h}, 100{ }^{\circ} \mathrm{C}$ ) and both hydrolysates were separated by using the methods described by Haug et al. [11]. The sodium salt of G-block hydrolysate was obtained as follows: G-block was suspended in ion-exchanged water, after which $0.2 \mathrm{M} \mathrm{NaOH}$ was added to the suspension with stirring. The $\mathrm{pH}$ of the solution was then increased to $\sim 7$ to produce the sodium salt of G-block. The addition of ethanol resulted in the formation of a white precipitate in the solution, which was then collected by centrifugation at $1900 \times \mathrm{g}$ for $5 \mathrm{~min}$. The pellet was washed three times with ethanol and then dried.

\section{Preparation of low molecular weight alginic acid (LM- ALG)}

LM-ALG was prepared as follows. Briefly, 2

$\mathrm{M} \mathrm{HCl}$ was gradually added to $30 \mathrm{~g}$ ULV-L3 solution (1\% $\mathrm{w} / \mathrm{w}$ ) with stirring and cooled with ice water. A deposit caused by coacervation occurred when the $\mathrm{pH}$ of the solution was reduced to below 3. The coacervate was centrifuged at $1900 \times \mathrm{xg}(2100$ model; Kubota Co., Tokyo, Japan) for $5 \mathrm{~min}$, washed once with ionexchanged water, washed three times with ethanol, and dried. The white block obtained was ground with a pestle to obtain alginic acid powder, which was sieved (mesh size, $75 \mu \mathrm{m}$ ) before use in subsequent experiments.

\section{FD preparation}

The additive was added to $10 \mathrm{~g}$ of the film base solution and agitated. The solution was thoroughly mixed by sonication and $3.0 \mathrm{~g}$ of each solution was poured into individual plastic Petri dishes (diameter: $54 \mathrm{~mm}$ ). After $24 \mathrm{~h}$ at $37{ }^{\circ} \mathrm{C}$, the circular films formed on each dish were transferred to a desiccator. In this method, $3 \mathrm{mg}$ MCZ was theoretically incorporated into each FD. Film formation was judged not to have occurred if a circular film was not obtained, if the film had cracks, or if the film could not be removed from the bottom of the dish.

\section{Film thickness and rheological properties}

Film thickness was measured at 10 points on each film by using a micrometer (CLM1-15QM; Mitutoyo, Kawasaki, Japan) with a set pressure of $0.5 \mathrm{~N}$. Measurements were collected from three films and the mean thickness was calculated for each type. The rheological properties of each film were determined by using a rheometer (SUN RHEO TEX SD-700\#; Sun Scientific Co., Tokyo, Japan) at room temperature. The film was fixed on a vial (inner diameter: $1.4 \mathrm{~mm}$; outer diameter: $18.8 \mathrm{~mm}$ ) by using a rubber band (Kyowa Co., Osaka, Japan) and probed by using a cylindrical adapter (diameter: $5.0 \mathrm{~mm}$ ). Stress and strain were measured at the point at which the adapter broke through the film. All tests were performed in triplicate.

\section{Measurement of MCZ content}

The high-performance liquid chromatography (HPLC) system (Hitachi Co., Tokyo, Japan) consisted of a pump (L-2130), UVdetector (L-2400), autosampler (L-2200), and chromate-integrator
(D-2500) connected to a packed column (150 $\mathrm{mm} \times 4.6 \mathrm{~mm}$, Cosmosil 5C18-MS-II, Nacalai Tesque, Kyoto, Japan). To determine the concentration of MCZ, HPLC was conducted at ambient temperature with an eluent that consisted of $10 \mathrm{mM} \mathrm{KH} 2 \mathrm{PO} 4$ and acetonitrile (1:4) at a flow rate of $1 \mathrm{ml} / \mathrm{min}$. The detection wavelength was $230 \mathrm{~nm}[12]$

\section{Solubility of MCZ}

The solubility of MCZ was measured in physiological saline containing an additive. MCZ was added to the test solution and shaken at $37{ }^{\circ} \mathrm{C}$ for $24 \mathrm{~h}$. The suspension was removed by using a pre-heated plastic syringe (Terumo Co., Tokyo, Japan) at $37{ }^{\circ} \mathrm{C}$ and filtered by using a syringe driven filter unit (Millex-HV, pore size: $0.45 \mathrm{~mm}$, Millipore Co., Danvers, MA, USA). The solution was diluted with physiological saline and injected onto the HPLC column.

\section{MCZ dissolution test}

Physiological saline preheated to $37{ }^{\circ} \mathrm{C}$ was added to the FD, placed in a plastic dish, and then the dish was shaken at 300rpm in a shaker incubator (SI-300; As One Co., Osaka, Japan) at $37{ }^{\circ} \mathrm{C}$. After 1, 3, 5, 10, 15, 20, 30, 45, and $60 \mathrm{~min}$, a $0.3 \mathrm{ml}$ aliquot of each solution was removed periodically by using a plastic syringe, after which $0.3 \mathrm{ml}$ of the test medium $\left(37^{\circ} \mathrm{C}\right)$ was added to maintain a constant volume. The sample solution was centrifuged $(7,700 \times \mathrm{g}$, 1min; H-1300; Kokusan Co., Saitama, Japan). Then, the solution was filtered through a syringe-driven filter unit (pore size: $0.45 \mathrm{~mm}$ ). Eighty-microliter aliquots of the filtered solution were placed into micro-test tubes $(1.5 \mathrm{ml})$ and $720 \mathrm{ml}$ methanol was added to precipitate the polysaccharide dissolved from the dosage form. The samples were mixed, centrifuged (7,700×g, 5min; H-1300; Kokusan Co., Saitama, Japan), and the supernatants were injected into the HPLC column. All tests were performed in triplicate.

\section{Results and Discussion}

FD incorporating MCZ was formed by pouring the film base solution into a Petri dish and evaporating the solvent. The addition of the hydrolysate to the solution affected FD formation. A circular film was obtained from the film base solution containing $0.1 \%$ G-block or M-block, with a thickness of approximately $70 \mu \mathrm{m}$, as shown in Figure 1 and Table 1. Similar FDs were also obtained in the case of a base solution containing $0.02 \%-1.0 \%$ LM-ALG. However, the film prepared with a solution containing 1\% G-block was cracked.

Table 1: Thickness of FDs prepared with 1.5\% Alg-Na containing alginate hydrolysate.

\begin{tabular}{|c|c|c|c|}
\hline Additive & \multicolumn{3}{|c|}{ Thickness ( mm) } \\
\hline No additives & 74 & \pm 4 & \pm 4 \\
\hline $0.10 \%$ & G-block & 74 & \pm 5 \\
\hline $0.30 \%$ & G-block & 70 & \pm 14 \\
\hline $0.50 \%$ & G-block & 83 & \pm 3 \\
\hline $0.10 \%$ & M-block & 76 & \pm 3 \\
\hline $0.30 \%$ & M-block & 73 & \pm 7 \\
\hline $0.50 \%$ & M-block & 74 & \\
\hline
\end{tabular}



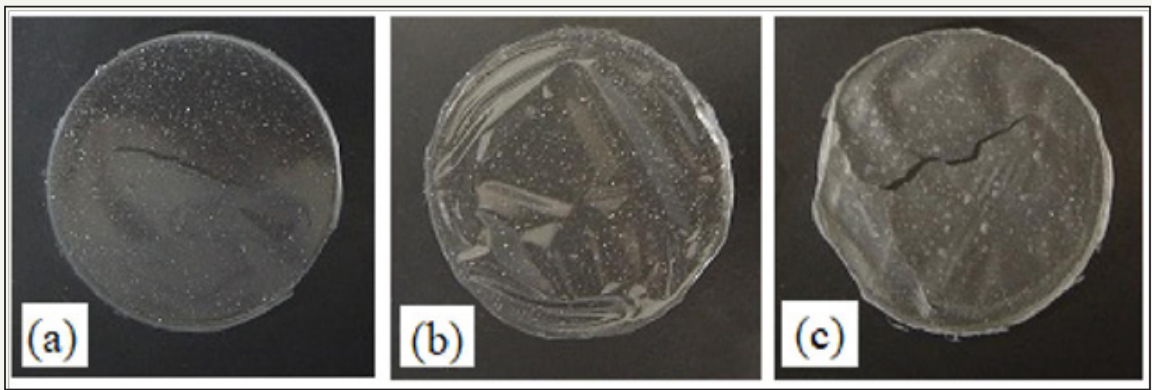

Figure 1: Pictures of FDs prepared with 1.5\% Alg-Na containing alginate hydrolysate. (a) $0.1 \%$ G-block. (b) $0.1 \%$ M-block. (c) $1 \%$ G-block.

As they are directly applied to the target region, FDs in oral candidiasis therapy should be easy to handle. The additive effect of the hydrolysate on the rheological properties of FDs is shown in Figure 2. FDs prepared with Alg-Na were soft and each FD had sufficient strength to be treated by hand, although the addition of $0.5 \%$ G-block in the film base reduced the resistance of FD to tearing.

FD prepared with Alg-Na immediately swelled in physiological saline at $37{ }^{\circ} \mathrm{C}$, which led to erosion. MCZ was gradually released from the FDs and the amount dissolved into the test solution at $60 \mathrm{~min}$ was $13 \% \pm 1 \%$ of the drug incorporated in the FD. The addition of hydrolysate to the film base accelerated the drug dissolution rate of FD, as shown in Figure 3. For example, 35\% of MCZ was dissolved over 5 min from FDs prepared with the base containing $0.1 \%$ G-block. Similar MCZ dissolution profiles were obtained by the addition of $0.3 \%$ or $0.5 \%$ G-block to FDs. This phenomenon was also observed in the case of FD modified with M-block.

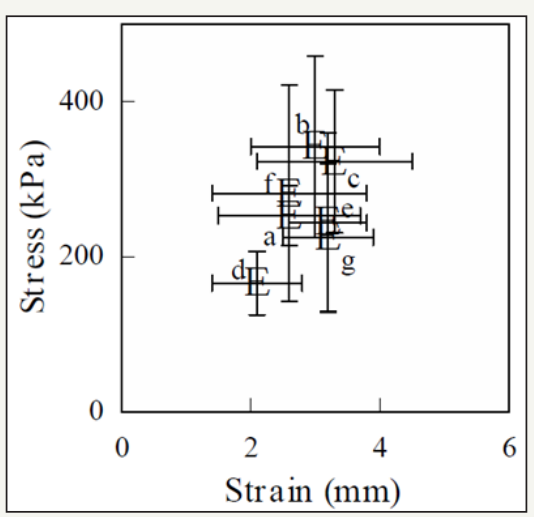

Figure 2: Rheological properties of FDs prepared with 1.5\% Alg-Na containing alginate hydrolysate. (a): additive free, (b): $0.1 \%$ G-block, (c): 0.3\% G-block, (d): 0.5\% G-block, (e):0.1\% M-block, (f): 0.3\% M-block, (g): 0.5\% M-block.

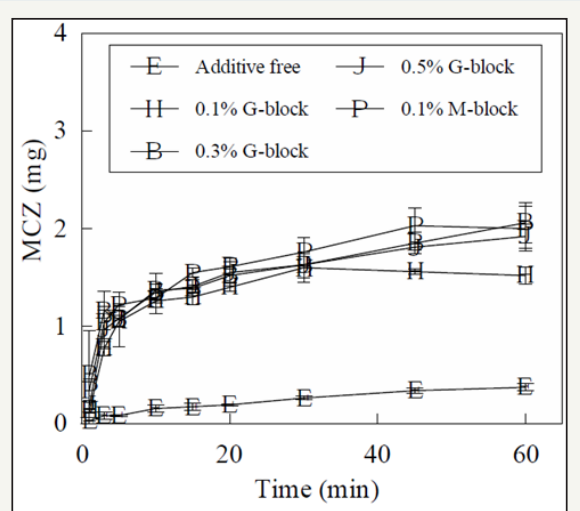

Figure 3: Dissolution profiles of MCZ from FDs prepared with 1.5\% Alg-Na containing alginate hydrolysate in physiological saline

The MCZ dissolution profiles from FDs prepared with base containing 0.01\%-0.05\% G-block are shown in Figure 4. The initial dissolution rate increased after the addition of G-block to the film base. In the case of FDs containing $0.05 \%$ G-block, $23 \% \pm 1 \%$ of the MCZ was dissolved after $5 \mathrm{~min}$. However, the addition of $0.1 \%$ G-block sodium salt to the base solution did not affect the drug 
dissolution profile. The acceleration of the drug dissolution rate was also recognized in FDs prepared with the film base containing
LM-ALG, although modification of the FDs with ULV-L3 did not affect the rate, as shown in Figure 5.

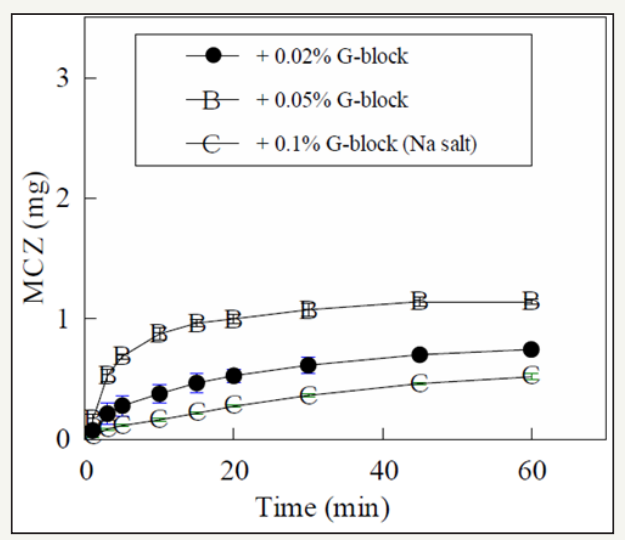

Figure 4: Effect of alginate hydrolysate concentration on $M C Z$ dissolution from FDs.

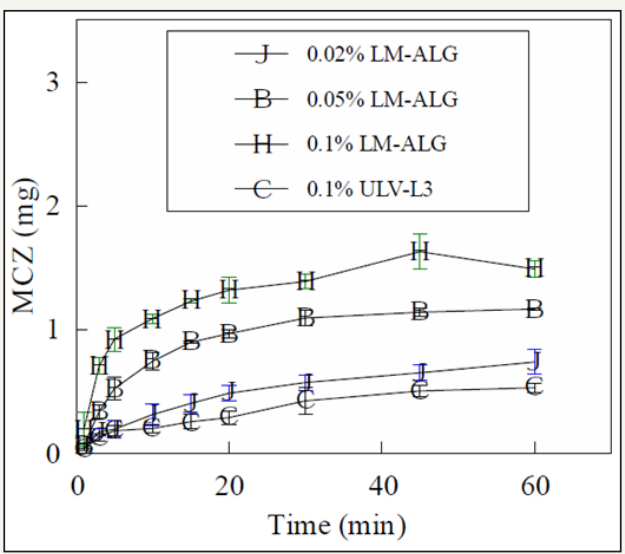

Figure 5: Effect of LM-ALG concentration on MCZ dissolution from FDs.

Table 2: Solubility of MCZ in physiological saline containing $0.1 \%$ additive at $37^{\circ} \mathrm{C}$.

\begin{tabular}{|c|c|}
\hline Additive & Solubility $(\mathbf{m g} / \mathbf{m L})$ \\
\hline No additives & 0.47 \\
\hline G-block & 0.4 \\
\hline M-block & 0.45 \\
\hline LM-ALG & 0.35 \\
\hline
\end{tabular}

These results suggested that the acceleration of MCZ dissolution from FD was attributable to the addition of a compound with a polycarboxylic acid, but not the sodium salt, to the film base. We already reported that the MCZ dissolution rate was accelerated by the modification of FD with additives, such as surface-active agents or cyclodextrins, which was able to increase the solubility of the drug in water [10]. The effect of additives on MCZ solubility in physiological saline at $37^{\circ} \mathrm{C}$ is shown in Table 2 . The solubility of MCZ was not affected by the addition of $0.1 \%$ G-block or M-block to the solution. In addition, the solubility was decreased by the addition of ULV-L3 compared to the solubility of an additive-free test solution.
In this study, FDs were prepared with Alg-Na containing the hydrolysates or LM-ALG and the drug release profiles from the dosage forms were investigated. Each material used for the preparation of FD was a species of alginic acid or the sodium salt and all were selected owing to their safety for oral administration. The film matrix prepared with these materials immediately swelled in a small amount of saliva and then disintegrated during the release of the incorporated drug. As FDs containing MCZ are administered in the oral cavity to act directly on the affected site, the drug dissolution rate in saliva is an important factor in the antifungal activity for the treatment of oral candidiasis. The MCZ dissolution rate can be controlled by the amount of additive in the base solution. FD prepared with Alg-Na and the hydrolysate is a promising candidate for the treatment of oral candidiasis.

\section{References}

1. Ghorbani Gorji E, Waheed A, Ludwig R, Toca-Herrera JL, Schleining G, et al. (2018) Complex coacervation of milk proteins with sodium alginate. J Agric Food Chem, doi: 10.1021/acs.jafc.7b03915.

2. Maestrelli F, Mura P, González-Rodríguez ML, Cózar-Bernal MJ, Rabasco AM, et al. (2017) Calcium alginate microspheres containing metformin 
hydrochloride niosomes and chitosomes aimed for oral therapy of type 2 diabetes mellitus. Int J Pharm 530(1-2): 430-439.

3. Fan L, Ge H, Zou S, Xiao Y, Wen H, et al. (2016) Sodium alginate conjugated graphene oxide as a new carrier for drug delivery system. Int J Biol Macromol 93(Pt A): 582-590.

4. Murata Y, Isobe T, Kofuji K, Nishida N, Kamaguchi R (2010) Preparation of fast dissolving films for oral dosage from natural polysaccharides. Materials 3(8): 4291-4299.

5. Murata Y, Sakano H, Maida C, Kofuji K (2017) Disintegration properties and drug release profiles of sodium alginate films modified with additives. Res Dev Material Sci 2(1): 1-4

6. Kassem AA, Issa DA, Kotry GS, Farid RM (2017) Thiolated alginate-based multiple layer mucoadhesive films of metformin forintra-pocket local delivery: in vitro characterization and clinical assessment. Drug Dev Ind Pharm 43(1): 120-131
7. Lewis MAO, Williams DW (2017) Diagnosis and management of oral candidosis. Br Dent J 223(9): 675-681.

8. George S, Balan A (2018) A potential side effect of oral topical steroids: Central serous chorioretinopathy. Indian J Dent Res 29(1): 107-108.

9. Hellfritzsch M, Pottegård A, Pedersen AJ, Burghle A, Mouaanaki F, et al. (2017) Topical antimycotics for oral candidiasis in warfarin users. Basic Clin Pharmacol Toxicol 120(4): 368-372.

10. Murata Y, Kofuji K, Nakano S, Kamaguchi R (2015) Cyclodextrin-modified film dosage forms for oral candidiasis treatment. Pharmacology \& Pharmacy 6: 247-253.

11. Haug A, Larsen B, Smidsrod O (1974) Uronic acid sequence in alginate from different sources. Carbohyd Res 32(2): 217-225.

12. Pershing LK, Corlett J, Jorgensen C (1994) In vivo pharmacokinetics and pharmacodynamics of topical ketoconazole and miconazole in human stratum corneum. Antimicrob Ag Chem 38(1): 90-95.
Creative Commons Attribution 4.0 International License

For possible submissions Click Here

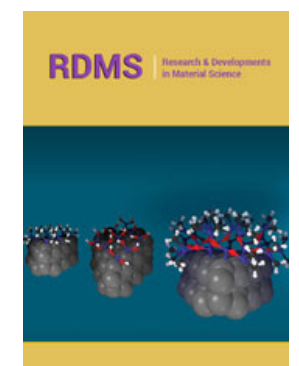

\section{Research \& Development in Material Science}

\section{Benefits of Publishing with us}

- High-level peer review and editorial services

- Freely accessible online immediately upon publication

- Authors retain the copyright to their work

- Licensing it under a Creative Commons license

- Visibility through different online platforms 